\title{
LETTERS
}

\section{Sarcolemma-localized nNOS is required to maintain activity after mild exercise}

\author{
Yvonne M. Kobayashi ${ }^{1,2,3,4}$, Erik P. Rader ${ }^{1,2,3,4}$, Robert W. Crawford ${ }^{1,2,3,4}$, Nikhil K. lyengar ${ }^{4}$, Daniel R. Thedens ${ }^{5}$, \\ John A. Faulkner ${ }^{7}$, Swapnesh V. Parikh ${ }^{4}$, Robert M. Weiss ${ }^{4}$, Jeffrey S. Chamberlain ${ }^{8}$, Steven A. Moore ${ }^{6}$ \\ \& Kevin P. Campbell ${ }^{1,2,3,4}$
}

Many neuromuscular conditions are characterized by an exaggerated exercise-induced fatigue response that is disproportionate to activity level. This fatigue is not necessarily correlated with greater central or peripheral fatigue in patients ${ }^{1}$, and some patients experience severe fatigue without any demonstrable somatic disease $^{2}$. Except in myopathies that are due to specific metabolic defects, the mechanism underlying this type of fatigue remains unknown ${ }^{2}$. With no treatment available, this form of inactivity is a major determinant of disability ${ }^{3}$. Here we show, using mouse models, that this exaggerated fatigue response is distinct from a loss in specific force production by muscle, and that sarcolemmalocalized signalling by neuronal nitric oxide synthase (nNOS) in skeletal muscle is required to maintain activity after mild exercise. We show that $n N O S$-null mice do not have muscle pathology and have no loss of muscle-specific force after exercise but do display this exaggerated fatigue response to mild exercise. In mouse models of nNOS mislocalization from the sarcolemma, prolonged inactivity was only relieved by pharmacologically enhancing the cGMP signal that results from muscle nNOS activation during the nitric oxide signalling response to mild exercise. Our findings suggest that the mechanism underlying the exaggerated fatigue response to mild exercise is a lack of contraction-induced signalling from sarcolemma-localized nNOS, which decreases cGMP-mediated vasomodulation in the vessels that supply active muscle after mild exercise. Sarcolemmal nNOS staining was decreased in patient biopsies from a large number of distinct myopathies, suggesting a common mechanism of fatigue. Our results suggest that patients with an exaggerated fatigue response to mild exercise would show clinical improvement in response to treatment strategies aimed at improving exercise-induced signalling.

To understand the molecular basis of the exercise-induced fatigue response, we studied genetically defined mouse models. We designed an integrative in vivo assay to test conscious mice, subjecting the mice to brief low-speed treadmill exercise followed by testing in an openfield activity chamber (see Methods). We first assessed two dystrophic mouse lines, $m d x$ (model for Duchenne muscular dystrophy) ${ }^{4}$ and Sgca-null (model for limb-girdle muscular dystrophy type $2 \mathrm{D}$ that is deficient for the gene encoding $\alpha$-sarcoglycan $(S g c a))^{5}$. In the absence of previous exercise, activity in these mice was indistinguishable from that of wild-type mice (Fig. 1a, b, and Supplementary Videos 1a-d). After a single trial of mild exercise, significant differences were observed (Fig. 1a, b, and Supplementary Videos $2 \mathrm{a}-\mathrm{d}$ ): the $m d x$ and $S g c a$-null mice showed a significant decrease in vertical activity.
The decrease in vertical activity among $m d x$ and $S g c a$-null mice did not correlate with differences in extensor digitorum longus (EDL)specific force measurements relative to those taken in C57BL/6 mice before exercise (Fig. 1c). Moreover, Sgca-null mice do not develop brain, heart or vascular pathology ${ }^{6}$, and they have muscle-force values similar to those of control mice ${ }^{7}$. Therefore, neither cardiac deficiency nor an inability to produce force was the cause of the postexercise inactivity in the $S g c a$-null mice. Because inflammation is a feature of dystrophinopathy ${ }^{4}$, chronic fatigue is associated with muscle pain, and chronic pain is associated with fatigue ${ }^{8}$, we treated $m d x$ mice with either deflazacort or ibuprofen. However, neither treatment resulted in improved post-exercise activity (Fig. 1d), suggesting that the inactivity occurring immediately after mild exercise in $m d x$ mice was not due to inflammation or pain. Overall, the results of our exercise-activity assay implied that the exaggerated fatigue response in these mice was not attributable to cardiac deficiency, inflammation, pain or lack of muscle force.

To test whether the exercise-induced inactivity in the $m d x$ and Sgca-null mice was due to the genetically determined structural defect in muscle, we assayed two mouse models in which the muscle pathology related to the specific dystrophin glycoprotein complex (DGC) defect is rescued-microdystrophin/ $m d x$ and MCKeSG/ Sgca-null. In microdystrophin/ $m d x$ mice (a model for mild Becker muscular dystrophy ${ }^{9}$ - the DGC has a mutated but functional dystrophin), microdystrophin is expressed in $m d x$ mouse muscle. In the MCKeSG/Sgca-null mice, $\varepsilon$-sarcoglycan is expressed in mouse muscle that is deficient for Sgca (Supplementary Fig. 1). Neither rescue strain showed pathological signs of muscular dystrophy, and the skeletal muscle DGC of both was recovered at the biochemical, structural and functional levels (refs 9, 10 and Supplementary Figs 1 and 2).

Despite having a structurally intact skeletal muscle DGC, microdystrophin $m d x$ mice experience a substantial decrease in activity after mild exercise, like their $m d x$ littermates (Fig. 1e). Because patients with Becker muscular dystrophy show profound fatigue after light exertion ${ }^{11}$, and loss of sarcolemma-localized nNOS serves as a diagnostic indicator of some forms of Becker muscular dystrophy ${ }^{12}$, a possible reason for the post-exercise inactivity is a loss of sarcolemma-localized nNOS. To test this possibility we probed for nNOS localization in microdystrophin/ $m d x$ skeletal muscle and found that the DGC generated in this rescue strain failed to recruit nNOS to the sarcolemma (Fig. 1e, inset). These data are in agreement with recent reports on microdystrophin expression in dystrophindeficient mouse models ${ }^{13}$. Moreover, the data suggest that exercise-



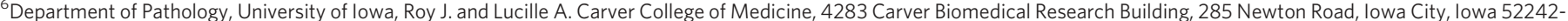
1101, USA. ${ }^{7}$ Department of Molecular and Integrative Physiology, University of Michigan, 2031 Biomedical Sciences Research Building, Ann Arbor, Michigan 48109-2200, USA.

${ }^{8}$ Department of Neurology, University of Washington School of Medicine, HSB, Room K243b, Seattle, Washington 98195-7720, USA.
} 

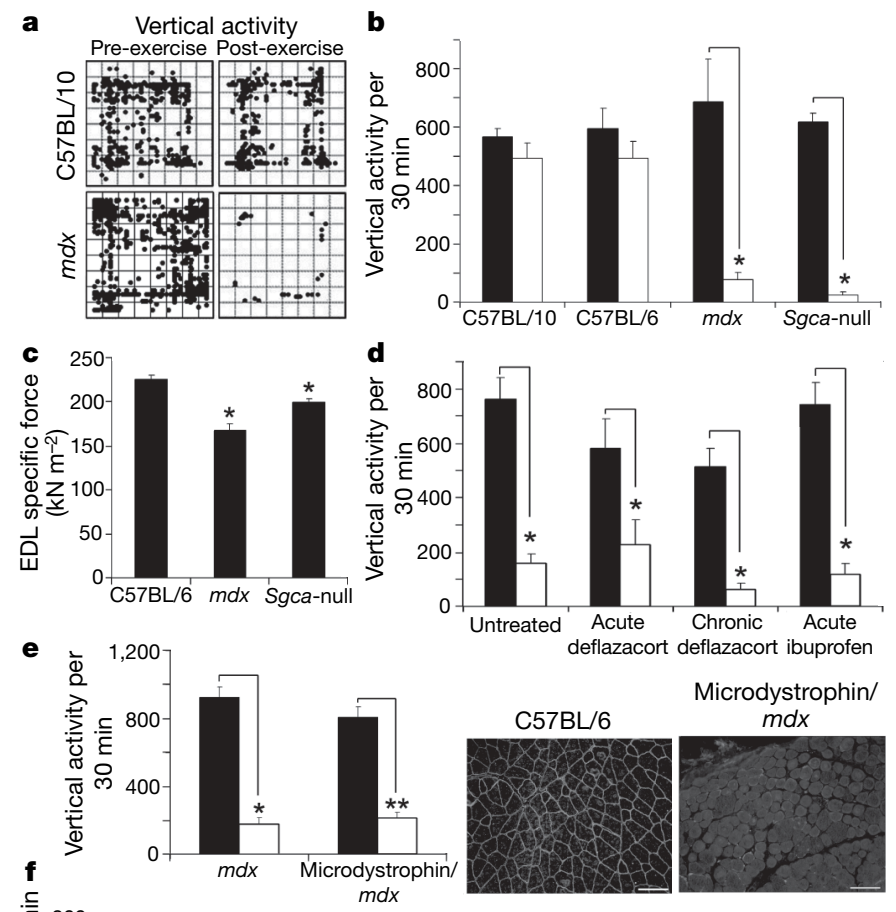

Microdystrophin/

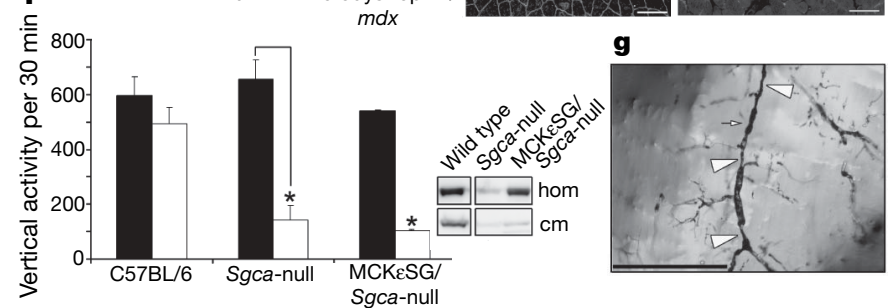

Figure 1 Loss of sarcolemma-localized nNOS leads to skeletal muscle vascular narrowings, decreased capillary perfusion and an exaggerated fatigue response after mild exercise in dystrophic and non-dystrophic mouse models. a, Representative vertical activity tracings of zone maps for $\mathrm{C} 57 \mathrm{BL} / 10$ and $m d x$ mice before and after exercise. $\mathbf{b}$, Quantified vertical activity before (filled columns) and after (open columns) exercise for C57BL/10, C57BL/6, $m d x$ and Sgca-null mouse strains ( $n=6$ for each strain). Asterisk, $P=0.012$. c, EDL muscle-specific force measurements from C57BL/6 $(n=6), m d x(n=4)$ and Sgca-null $(n=4)$ mice. Asterisk, $P<0.05$. d, Pre-exercise (filled columns) and post-exercise (open columns) vertical activity in untreated $(n=7)$ and anti-inflammatory treated $m d x$ mice, acutely $(n=4)$ or chronically $(n=4)$ with deflazacort or acutely with ibuprofen $(n=5)$. Asterisk, $P<0.003$. e, Left panel: quantified pre-exercise (filled columns) and post-exercise (open columns) vertical activity for microdystrophin $/ m d x$ mice $(n=6)$ and their $m d x$ littermates $(n=4)$. Asterisk, $P=0.005$; two asterisks, $P<0.0001$. The right panels show representative immunofluorescence images of nNOS detection in the gastrocnemius muscles from $\mathrm{C} 57 \mathrm{BL} / 6$ and microdystrophin $/ m d x$ mice. f, Quantified pre-exercise (filled columns) and post-exercise (open columns) vertical activity for MCKeSG/Sgca-null mice $(n=6)$ and their Sgca-null littermates $(n=6)$. Asterisk, $P<0.0001$. Inset: immunoblot detection of total nNOS from homogenates (hom), and crude skeletal muscle membranes $(\mathrm{cm}) . \mathbf{g}$, Representative Microfil image of skeletal muscle vessels of MCKESG/Sgca-null mice after exercise-large arrowheads mark extended areas of vascular narrowing; the small arrow marks a shorter stretch of radial vascular narrowing. Error bars indicate s.e.m.

induced inactivity in the microdystrophin $/ m d x$ mice is not caused directly by a structurally defective muscle DGC, and that loss of sarcolemmal nNOS does not negatively affect muscle contractility. Thus, sarcolemmal nNOS seems to act at the level of post-exercise activity.

In contrast to the microdystrophin/ $m d x$ mice, MCKeSG/Sgca-null mice have structurally intact DGC in the brain and the vasculature, but express $\varepsilon$-sarcoglycan instead of $\alpha$-sarcoglycan in the DGC of muscle. Our exercise-activity assay showed that post-exercise activity in the MCKeSG/Sgca-null mice was substantially decreased relative to that in C57BL/6 mice but similar to that in Sgca-null and $m d x$ mice (Fig. 1b, f). Because the microdystrophin-containing DGC failed to recruit nNOS, we speculated that the MCKESG/Sgca-null mice would also fail to localize nNOS to the sarcolemma. Indeed, although total nNOS levels in muscle homogenates from MCKeSG/Sgca-null mice were similar to those in the wild type, nNOS from the rescue model failed to purify together with the $\varepsilon$-sarcoglycan-containing DGC in the membrane preparation (Fig. 1f, inset). Taken together, these results are compatible with the notion that the exaggerated fatigue response is not directly related to a structurally defective muscle DGC or to muscle weakness, but rather to a failure in the sarcolemmal localization of nNOS.

Because sarcolemma-localized nNOS is crucial for maintaining vasomodulation to contracting muscles ${ }^{14}$, we tested whether communication from skeletal muscle to the local blood supply is deficient after mild exercise by perfusing MCK\&SG/Sgca-null mouse arteries before or after exercise with Microfil and examined the skeletal muscle vasculature (Fig. 1g). We identified vascular narrowings of various lengths along the arteries that feed the skeletal muscles in the post-exercise samples only, and also noted the lack of perfusion of capillaries. The $m d x$ and microdystrophin $/ m d x$ mice similarly showed skeletal muscle vascular narrowings only after exercise and also a lack of perfusion of capillaries (Supplementary Fig. 3c and data not shown). This phenotype is consistent with inefficient contraction-induced muscle nNOS signalling to local blood vessels. Overall, these data imply that loss of sarcolemma-localized nNOS causes deficient exercise-induced vasomodulation in skeletal muscle, and that these lead to prolonged inactivity after mild exercise.

To directly examine the contribution of NO generated by endothelial NOS (eNOS) or nNOS to the exaggerated fatigue response, we tested both $n N O S$-null and eNOS-null mice in our exercise-activity assay. Mice deficient for nNOS express normal levels of the DGC components at the sarcolemma and have histologically normal mus$\mathrm{cle}^{15-17}$. Reports suggest that both mouse strains have defective vasoregulation ${ }^{18,19}$; however, $m d x$ and $n N O S$-null mice have a normal $\alpha$-adrenergic vasoconstrictive response to exercise ${ }^{20}$. Vertical preexercise activities were similar in eNOS-null, $n N O S$-null and C57BL/6 mice, suggesting that the loss of either NOS does not affect mouse activity (Fig. 2a). After exercise, however, $n N O S$-null vertical activity decreased significantly (Fig. 2a). Serum creatine kinase levels before and after exercise for each of the NOS-null mice were similar to those in C57BL/ 6 mice and low compared with $m d x$ mice (Fig. 2b), and there were no signs of muscle pathology in sections from $n N O S$ null quadriceps muscle (Supplementary Fig. 4b), suggesting that muscle damage and necrosis were not the causes of the post-exercise inactivity. We then tested whether post-exercise muscle contractility affected the ability of C57BL/6 and $n N O S$-null skeletal muscle to produce force after mild exercise. We found that the specific force of EDL muscles after exercise was not significantly affected in $n N O S$ null muscle in comparison with C57BL/6 muscle (Supplementary Fig. 4c). Because lack of muscle contractility was not causing the inactivity in the $n N O S$-null mice after exercise, we checked whether NOS-null mice had post-exercise skeletal muscle vascular narrowings and lack of capillary perfusion similar to those in the dystrophic and rescue mice. Microfil perfusion of arteries from NOS-null mice before and after exercise revealed the lack of capillary perfusion and also the presence of vascular narrowings only in post-exercise $n N O S$-null skeletal muscle (Fig. 2c). We also found that treating wild-type mice with either the nNOS-specific inhibitor 3-bromo-7nitroindazole or the vasoconstrictor sarafotoxin $6 \mathrm{c}$ caused post-exercise inactivity (Fig. 2d). These findings suggest that a deficiency of sarcolemma-localized nNOS causes exercise-induced narrowing of the vasculature that feeds active muscles after exercise, thereby promoting prolonged inactivity after mild exercise.

To test whether the vascular effect on post-exercise activity was from NO or was downstream of the NO signal, we bypassed 
sarcolemmal nNOS signalling for decreasing vasoconstriction by treating $m d x$ mice with a panel of pharmacological agents that promote vasodilation; we found that the exaggerated fatigue response was alleviated only by treatment with a phosphodiesterase (PDE) 5A inhibitor (Supplementary Fig. 6), suggesting that the fatigue that we saw depended on cGMP, which acts downstream of NO production. $\mathrm{PDE}$ activity in $m d x$ mice is $2-6$-fold higher than in C57BL/10 mice ${ }^{21}$, which is consistent with the elevated PDE activity in human muscular disorders ${ }^{18,21,22}$. We treated $n N O S$-null, MCKeSG/Sgca-null and $m d x$ mice with PDE5A inhibitors and tested them in our exercise-activity assay; we found that the treated MCKeSG/Sgca-null and $m d x$ mice showed an increase in post-exercise activity (Fig. 2e and Supplementary Fig. 7a-d). Because inhibition of PDE5A had no effect on activity before exercise, our results suggest that PDE5A inhibition is alleviating the exaggerated fatigue response by enhancing the cGMP signal produced by contraction-induced nNOS stimulation. Although downstream effectors of cGMP are numerous
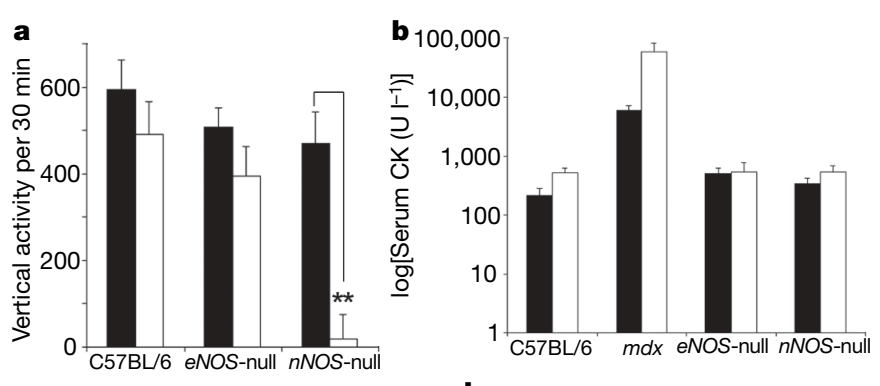

c
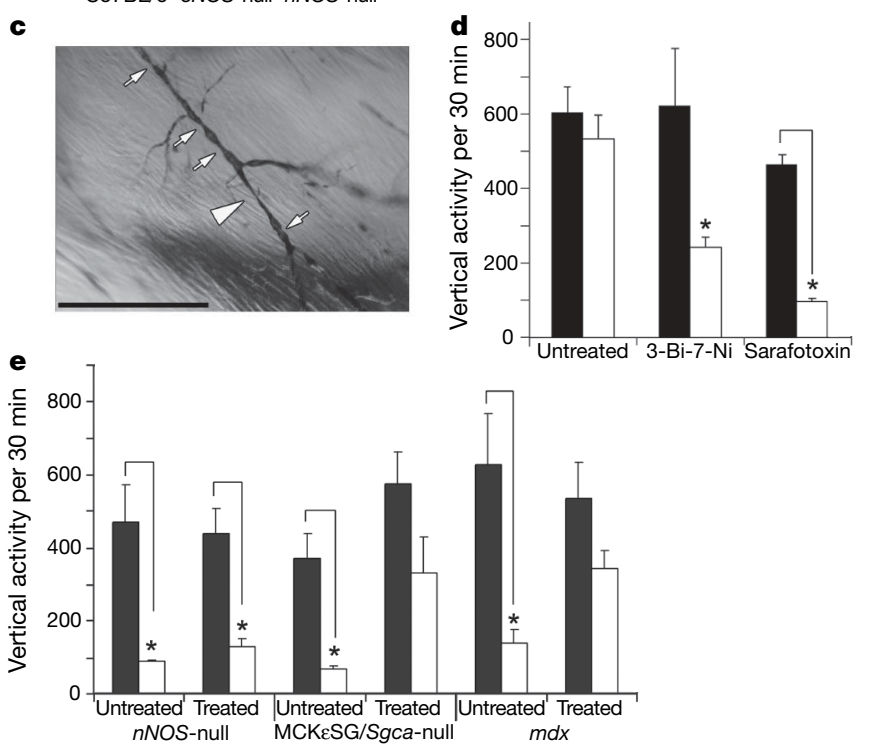

Figure 2 | Enhancing the cGMP signal resulting from muscle nNOS activation decreases the exaggerated fatigue response to mild exercise. a, Comparison of vertical activity before (filled columns) and after (open columns) exercise between C57BL/6, eNOS-null and $n N O S$-null mice $(n=6$ for each). Two asterisks, $P<0.001$. b, Serum creatine kinase (CK) levels before (filled columns) and after (open columns) exercise in C57BL/6 $(n=6)$, eNOS-null $(n=4)$ and $n N O S$-null $(n=6)$ mice, compared with $m d x$ mice $(n=6)$.c, Representative Microfil image of $n N O S$-null quadriceps skeletal muscle arteries after exercise - the large arrowhead marks the extended area of vascular narrowing; small arrows mark shorter areas of radial vascular narrowing. Scale bar, $100 \mu \mathrm{m}$. d, Pre-exercise (filled columns) and post-exercise (open columns) vertical activities in untreated wild-type (C57BL/6 and C57BL/10) mice $(n=4)$, compared with 3-B-7-Ni-treated wild-type mice $(n=3)$ and sarafotoxin-treated wild-type mice $(n=4)$. Asterisk, $P<0.01$. e, Quantified pre-exercise (filled columns) and postexercise (open columns) activity with and without treatment with PDE5A inhibitor, in $n N O S$-null $(n=4), \mathrm{MCK \varepsilon SG} / S g c a-n u l l(n=4)$ and $m d x$ mice $(n=6)$. Asterisk, $P<0.0001$. Pre-exercise and post-exercise vertical activity error bars are s.e.m.

and divergent ${ }^{23}$, the half-life of cGMP can be affected by the activity of PDE5A. Our data indicate that the elevated PDE activity in extracts from $m d x$ mice could be PDE5A activity, and that PDE activity could also be elevated in the rescue mouse models we tested.

Our data suggest that the local resistance of arterioles that perfuse sarcolemmal nNOS-deficient muscles increases during exercise and that the lack of activity after mild exercise will lead to muscle oedema. We examined blood flow before and after exercise with laser Doppler imaging and found that blood flow in $m d x$ mice failed to increase as it did in C57BL/6 mice (Fig. 3a and Supplementary Fig. 7a), but treatment of $m d x$ mice with a PDE5A inhibitor alleviated this defect (Fig. 3b) and increased muscle capillary perfusion (Fig. 3c). Given that insufficient relief of local vasoconstriction in active muscles can lead to muscle oedema ${ }^{24}$, and that boys with Duchenne muscular dystrophy show muscle oedema ${ }^{25}$, we looked for changes in water compartmentalization and dynamics in the hindleg muscles of $n N O S$-null, C57BL/10 and $m d x$ mice before and after exercise by using spin-spin relaxation time $\left(T_{2}\right)$-magnetic resonance imaging. The $n N O S$-null mice did not have muscle damage or loss of contractility after exercise (Fig. 2b and Supplementary Fig. 4b, c), nor did they have muscle oedema (Supplementary Fig. 8a), suggesting that their lack of muscle damage prevents water accumulation in the tissue. Similarly, C57BL/10 mice showed little to no oedema in hindleg muscles after exercise $(0.70 \pm 0.50 \%$ (mean \pm s.e.m. $)$ ) (Fig. $3 \mathrm{f}$ and

a

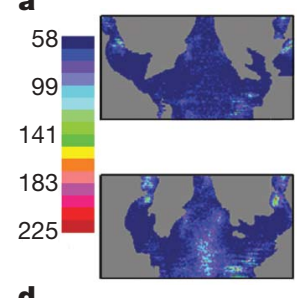

d

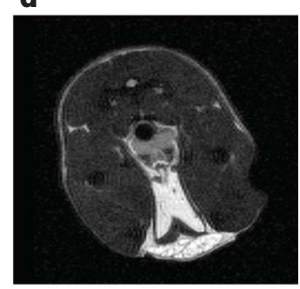

$\mathbf{f}-30$

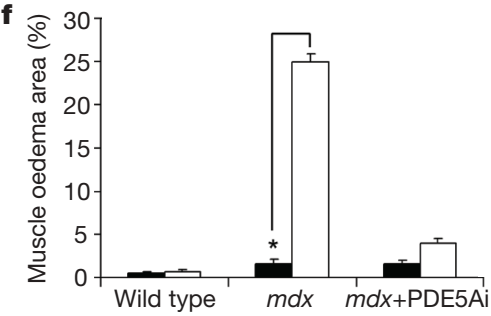

Figure 3 Treatment with PDE5A inhibitor improves exercised-induced vasomodulation and decreases exercise-induced oedema in $\mathbf{m d x}$ mice. a, Representative images of coronal laser Doppler analysis of blood flow in $m d x$ mice before (top) and after (bottom) exercise $(n=3)$. $\mathbf{b}$, Coronal laser Doppler analysis of blood flow in $m d x$ mice, before (top) and after (bottom) exercise, treated with PDE5A inhibitor before exercise $(n=3)$.

c, Representative Microfil image of quadriceps skeletal muscle arteries after exercise from $m d x$ mice treated with PDE5A inhibitor before exercise $(n=3$; scale bar, $100 \mu \mathrm{m})$. d, e, Representative axial views, by magnetic resonance imaging, of $m d x$ hindlimb muscles before (left) and after (right) exercise $(n=5)(d)$ and hindlimb muscles after exercise of $m d x$ mice treated with PDE5A inhibitor before exercise $(n=5)(\mathbf{e})$. White arrowheads mark areas of increased water compartmentalization. $\mathbf{f}$, Percentage muscle oedema area before (filled columns) and after (open columns) exercise, and with or without treatment with PDE5A inhibitor, in $m d x$ mice compared with that of the wild type. (Wild type and $m d x, n=3$; $m d x$ plus PDE5A inhibitor, $n=5$; error bars are s.e.m.) Asterisk, $P<0.001$. 


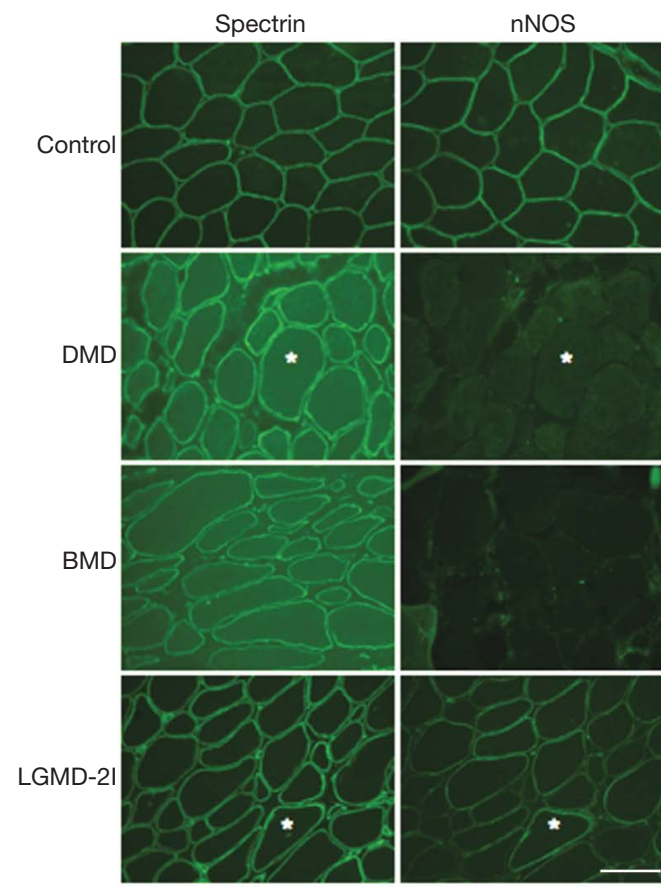

Figure 4 | nNOS levels in sarcolemma are decreased in human muscle diseases. Representative immunofluorescent staining in various human muscle diseases: primary dystrophinopathies, Duchenne and Becker muscular dystrophy (DMD and BMD, respectively); in several forms of limb-girdle muscular dystrophy (LGMD); in two congenital muscular

Supplementary Fig. 8b). However, hindleg muscles of $m d x$ mice consistently showed significant changes in tissue hydration after exercise $(25.0 \pm 2.45 \%$ (mean \pm s.e.m. $)$ ) (Fig. $3 \mathrm{~d}, \mathrm{f})$ that were indicative of exercise-induced muscle oedema. The water accumulation observed in the $m d x$ muscles is probably due to a combination of the increased local resistance in the arterioles that feed the active leg muscles and of muscle fibre fragility and damage. We also consistently found that treatment with PDE5A inhibitor significantly decreased exercise-induced muscle oedema in $m d x$ mice (3.99 $\pm 0.82 \%$ (mean \pm s.e.m.); Fig. 3e, f). Overall, our data imply that treatment with PDE5A inhibitor can relieve the post-exercise inactivity by normalizing PDE activity, thereby allowing the available NO derived from muscle nNOS to signal for cGMP-dependent vasodilation in active muscle; treatment with PDE5A inhibitor decreases muscle damage in $m d x$ mice by improving modulation of vascular activity in active muscle, thus preventing muscle oedema from exacerbating the muscle damage that occurs during the contraction of dystrophic muscle.

Because more than $60 \%$ of all patients with neuromuscular disease suffer from severe fatigue ${ }^{2}$, we tested for nNOS localization to the sarcolemma in biopsies of patients representing different myopathic disorders (Fig. 4, Supplementary Fig. 9 and Supplementary Table 1). In most myopathic biopsies assessed, sarcolemma-localized nNOS was either reduced or not detected, implying that many myopathic disorders may share a mechanism that results in severe exerciseinduced fatigue. Although increased fatigability inevitably occurs in patients with muscle weakness ${ }^{1}$, our mouse data imply that the exercise-induced inactivity is distinct from muscle weakness and that loss of sarcolemma-localized nNOS leads to an exaggerated fatigue response to mild exercise.

Our mouse data show that decreased or mislocalized skeletal muscle nNOS exacerbates the fatigue experienced after mild exercise because the normal contraction-induced cGMP-dependent attenuation of local vasoconstriction fails to occur, and that this failure causes vascular narrowing in muscles after exercise. In addition, our data from $m d x$ mice suggest that, as a result of nNOS mislocalization

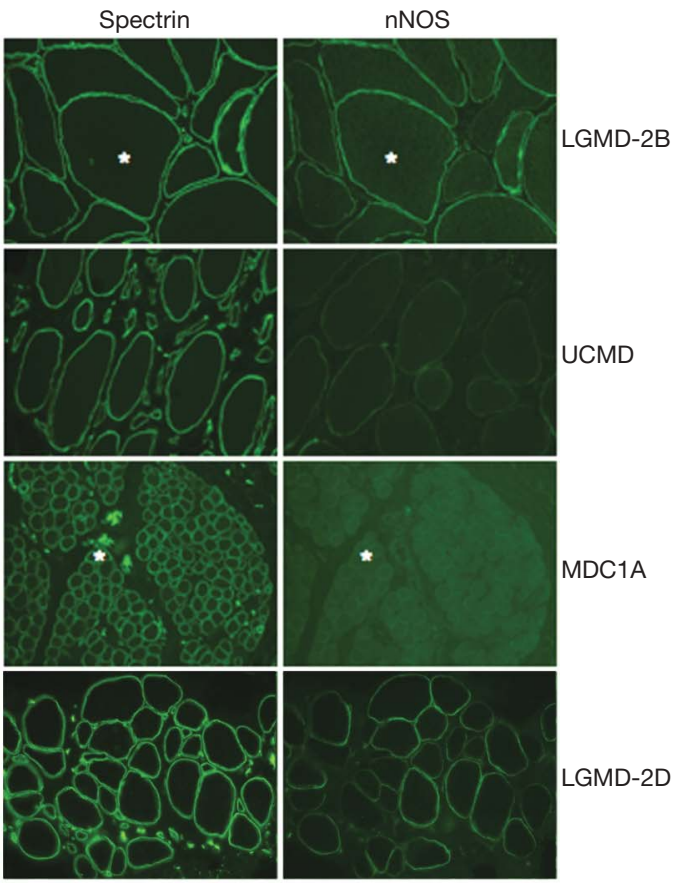

dystrophies (CMD) caused by mutations in extracellular matrix proteins (Ullrich CMD (UCMD), collagen VI and merosin-deficient CMD (MDC1A). Asterisks mark the same muscle fibres in some of the adjacent panels. Scale bar, $100 \mu \mathrm{m}$.

and increased PDE activity ${ }^{10,18,21}$, signalling for increased vasodilation to active muscle is deficient, causing muscle oedema. This, in turn, contributes to increased muscle damage as well as profound postexercise debility. Although the exact mechanism that leads to the inactivity after mild exercise has not been reduced to a single beginning and end pathway, our data suggest that contraction-induced cGMP-dependent attenuation of local vasoconstriction is pivotal in this mechanism. These findings could lead to a better understanding of muscle fatigue under other physiological conditions in which muscle nNOS expression, localization or activity is affected.

\section{METHODS SUMMARY}

Mouse models. Animal care and procedures were approved and performed in accordance with the standards set forth by the National Institutes of Health and the University of Iowa Animal Care and Use Committee.

Treadmill exercise and activity monitoring. Animals were mildly exercised with an adjustable variable-speed belt treadmill from AccuPacer. Activity based on ambulatory behaviour was assessed in an open-field test.

Received 16 January; accepted 29 August 2008.

Published online 26 October 2008.

1. Schillings, M. L. et al. Experienced and physiological fatigue in neuromuscular disorders. Clin. Neurophysiol. 118, 292-300 (2007).

2. Zwarts, M. J., Bleijenberg, G. \& van Engelen, B. G. Clinical neurophysiology of fatigue. Clin. Neurophysiol. doi:10.1016/j.clinph.2007.09.126 (2007).

3. Kalkman, J. S., Schillings, M. L., Zwarts, M. J., van Engelen, B. G. \& Bleijenberg, G The development of a model of fatigue in neuromuscular disorders: A longitudinal study. J. Psychosom. Res. 62, 571-579 (2007).

4. Radley, H. G., De Luca, A., Lynch, G. S. \& Grounds, M. D. Duchenne muscular dystrophy: focus on pharmaceutical and nutritional interventions. Int. J. Biochem Cell Biol. 39, 469-477 (2007).

5. Duclos, F. et al. Progressive muscular dystrophy in $\alpha$-sarcoglycan-deficient mice. J. Cell Biol. 142, 1461-1471 (1998).

6. Ozawa, E., Mizuno, Y., Hagiwara, Y., Sasaoka, T. \& Yoshida, M. Molecular and cell biology of the sarcoglycan complex. Muscle Nerve 32, 563-576 (2005).

7. Consolino, C. M. et al. Muscles of mice deficient in $\alpha$-sarcoglycan maintain large masses and near control force values throughout the life span. Physiol. Genomics 22, 244-256 (2005).

8. Yokoyama, T., Lisi, T. L., Moore, S. A. \& Sluka, K. A. Muscle fatigue increases the probability of developing hyperalgesia in mice. J. Pain 8, 692-699 (2007). 
9. Harper, S. Q. et al. Modular flexibility of dystrophin: implications for gene therapy of Duchenne muscular dystrophy. Nature Med. 8, 253-261 (2002).

10. Imamura, M., Mochizuki, Y., Engvall, E. \& Takeda, S. I. $\varepsilon$-Sarcoglycan compensates for lack of $\alpha$-sarcoglycan in a mouse model of limb-girdle muscular dystrophy. Hum. Mol. Genet. 14, 775-783 (2005).

11. Phillips, B. A. \& Mastaglia, F. L. Exercise therapy in patients with myopathy. Curr. Opin. Neurol. 13, 547-552 (2000)

12. Torelli, S. et al. Absence of neuronal nitric oxide synthase (nNOS) as a pathological marker for the diagnosis of Becker muscular dystrophy with rod domain deletions. Neuropathol. Appl. Neurobiol. 30, 540-545 (2004).

13. Judge, L. M., Haraguchiln, M. \& Chamberlain, J. S. Dissecting the signaling and mechanical functions of the dystrophin-glycoprotein complex. J. Cell Sci. 119, 1537-1546 (2006).

14. Thomas, G. D., Shaul, P. W., Yuhanna, I. S., Froehner, S. C. \& Adams, M. E. Vasomodulation by skeletal muscle-derived nitric oxide requires $\alpha$-syntrophinmediated sarcolemmal localization of neuronal nitric oxide synthase. Circ. Res. 92, 554-560 (2003)

15. Chao, D. S., Silvagno, F. \& Bredt, D. S. Muscular dystrophy in $m d x$ mice despite lack of neuronal nitric oxide synthase. J. Neurochem. 71, 784-789 (1998).

16. Crosbie, R. H. et al. $m d x$ muscle pathology is independent of nNOS perturbation. Hum. Mol. Genet. 7, 823-829 (1998).

17. Suzuki, N. et al. NO production results in suspension-induced muscle atrophy through dislocation of neuronal NOS. J. Clin. Invest. 117, 2468-2476 (2007).

18. Asai, A. et al. Primary role of functional ischemia, quantitative evidence for the two-hit mechanism, and phosphodiesterase-5 inhibitor therapy in mouse muscular dystrophy. PLOS ONE 2, e806 (2007).

19. Huang, P. L. et al. Hypertension in mice lacking the gene for endothelial nitric oxide synthase. Nature 377, 239-242 (1995).

20. Thomas, G. D. et al. Impaired metabolic modulation of $\alpha$-adrenergic vasoconstriction in dystrophin-deficient skeletal muscle. Proc. Natl Acad. Sci. USA 95, 15090-15095 (1998).
21. Bloom, T. J. Age-related alterations in cyclic nucleotide phosphodiesterase activity in dystrophic mouse leg muscle. Can. J. Physiol. Pharmacol. 83, 1055-1060 (2005).

22. Bloom, T. J. Cyclic nucleotide phosphodiesterase isozymes expressed in mouse skeletal muscle. Can. J. Physiol. Pharmacol. 80, 1132-1135 (2002).

23. Kass, D. A., Champion, H. C. \& Beavo, J. A. Phosphodiesterase type 5: expanding roles in cardiovascular regulation. Circ. Res. 101, 1084-1095 (2007).

24. Persson, J., Ekelund, U. \& Grande, P. O. Endogenous nitric oxide reduces microvascular permeability and tissue oedema during exercise in cat skeletal muscle. J. Vasc. Res. 40, 538-546 (2003)

25. Marden, F. A., Connolly, A. M., Siegel, M. J. \& Rubin, D. A. Compositional analysis of muscle in boys with Duchenne muscular dystrophy using MR imaging. Skeletal Radiol. 34, 140-148 (2005).

Supplementary Information is linked to the online version of the paper at www.nature.com/nature.

Acknowledgements We thank M. Anderson and M. Henry for comments, and M. M. Kilburg, K. Uppal, B. J. Steinmann and S. Watkins and members of the Campbell laboratory for scientific contributions. This work was supported in part by a Paul D. Wellstone Muscular Dystrophy Cooperative Research Center Grant. Y.M.K. was supported by grants from the University of lowa Cardiovascular Interdisciplinary Research/ National Research Service Award (NRSA) Fellowship, from an individual NRSA Fellowship from the National Institute of Arthritis and Musculoskeletal and Skin Diseases, from the National Institutes of Health (NIH), and from a Senator Paul D. Wellstone Fellowship. E.P.R. was supported by a Muscular Dystrophy Association Development Grant. R.M.W. was supported by the NIH. K.P.C. is an investigator of the Howard Hughes Medical Institute.

Author Information Reprints and permissions information is available at www.nature.com/reprints. Correspondence and requests for materials should be addressed to K.P.C. (kevin-campbell@uiowa.edu). 